\title{
COHERENT AND SATURATED COLLECTIONS
}

\author{
BY \\ G. T. WHYBURN
}

1. Introduction. The concepts with which this paper is concerned are of considerable significance in topology and occur in some form in many other branches of mathematics. Coherence is a sort of connectedness and comprises the association together of a set of things in some concrete relationship which persists in the face of other admissible elements or sets or forces which may be present. Saturation is meant to embody the concept of a set being as large or as full as possible without losing certain essential properties with which the set is endowed. This includes various sorts of maximal sets and, by contrast, is related to minimal sets.

Connectedness itself represents one of the strongest types of cohesion. For if a set $M$ is connected, it holds together irrespective of other elements present in the sense that however it be broken into two non-empty subsets $M_{1}$ and $M_{2}$, one of them will contain a point or a limit point of the other. Also, a component $C$ of a given set $K$ is a subset which is saturated in $K$ relative to the property of being connected, that is, $C$ has this property but if anything is added to $C$ in $K$, the property of connectedness is lost. Similarly, a cyclic element $E$ of a locally connected continuum $M$ is a set saturated in $M$ relative to the property of being connected and having no cut point, that is, $E$ has these properties, but if anything is added to $E$, at least one of the properties will be lost.

More specifically, the background for the kind of collections of sets I shall deal with is to be found in the notion of a so-called non-separated collection of subsets of a connected metric space $M$, together with the associated non-alternating transformations generated by such collections, that is, collections $G$ of subsets of $M$ which do not separate each other in $M$ so that if $X, Y$ belong to $G$, then we cannot separate $M-X$ into two separated sets each of which contains a point of $Y$. These ideas were developed in papers published in 1930 and 1934 respectively $[1,2]\left({ }^{1}\right)$ and since then have been broadened and extended in several different directions by Hall and Schweigert [3], Wardwell [4], Wallace [5], White [6] and several others.

The main objective of the present paper will be to develop a general theory of coherence and saturation of collections of subsets of a connected separable metric space $M$ which embraces many of the previous studies; and to show how in a very general setting such collections can be made to generate a

Presented to the Society, April 4, 1942; received by the editors September 12, 1944.

(1) Numbers in brackets refer to the Bibliography at the end of the paper. 
complete upper semi-continuous decomposition of the space $M$ and thus in turn generate a mapping on $M$ of a particularly useful nature which will be non-alternating in a sense corresponding to the degree of coherence of the given collection.

2. Definitions. Setting. Our considerations will be confined to a separable metric space designated by $M ; G$ will denote an arbitrary collection of subsets of $M$; and $\alpha$ will stand for one of the numbers $1,2, \cdots, \omega, \boldsymbol{N}_{0}, c$. The latter numbers are all cardinals except $\omega$, which is a symbol inserted to stand for an arbitrary finite cardinal. Thus in what follows the case $\alpha=\omega$ is to be interpreted as meaning that the sets referred to are finite in all cases although they may have a varying number of elements.

A closed set which is the sum of not more than $\alpha$ elements of $G$ will be called an $F(G, \alpha)$.

If $x, y \in M, x$ is said to be $(G, \alpha)$-conjugate to $y$, written $x(G, \alpha) y$, provided that no $F(G, \alpha)$ separates $x$ and $y$ in $M$.

A collection $G^{\prime}$ of subsets of $M$ is said to be: (a) $(G, \alpha)$-coherent provided $x, y \in g^{\prime} \in G^{\prime}$ implies $x(G, \alpha) y$; (b) $(G, \alpha)$-saturated provided that for $x \in g^{\prime} \in G^{\prime}$, $x(G, \alpha) y$ implies $y \in g^{\prime}$; (c) $(G, \alpha)$-determined provided that for $x \in g^{\prime} \in G^{\prime}$, $x(G, \alpha) y$ is equivalent to $y \in g^{\prime}$.

To illustrate, let $M$ be the set $1 \leqq \rho \leqq 2$ in a plane with polar coordinates. Let $G^{\prime}$ denote the collection of sets $\theta=2 \pi t, 1 \leqq \rho \leqq 2$ for $0 \leqq t \leqq 1$, and let $G$ be the subcollection of $G^{\prime}$ corresponding to rational $t$. Then for $\alpha=2$, both $G$ and $G^{\prime}$ are $(G, \alpha)$-determined [and thus $(G, \alpha)$-coherent and $(G, \alpha)$-saturated, since clearly these two together are the same as $(G, \alpha)$-determined]. For $\alpha=1$, these sets are not saturated, though of course they are coherent. On the other hand, if $G^{\prime}$ is the collection of sets $\rho=s$ for $1 \leqq s \leqq 2$ and $G$ is the subcollection corresponding to rational $s$, then for $\alpha=1$ both $G$ and $G^{\prime}$ are $(G, \alpha)$-determined.

It may be remarked that if $\alpha<\beta,(G, \alpha)$-saturation implies $(G, \beta)$-saturation and $(G, \beta)$-coherence implies $(G, \alpha)$-coherence for any $G$. Also if $G_{2} \supset G_{1}$, $\left(G_{1}, \alpha\right)$-saturation implies $\left(G_{2}, \alpha\right)$-saturation and $\left(G_{2}, \alpha\right)$-coherence implies $\left(G_{1}, \alpha\right)$-coherence for any $\alpha$.

3. Basic properties. We proceed with the development of the fundamental properties associated with these notions. relation.

(3.1) Theorem. If $G$ is $(G, \alpha)$-saturated, $(G, \alpha)$-conjugacy is an equivalence

For obviously $x(G, \alpha) x$ for any $x \in M$ and $x(G, \alpha) y$ implies $y(G, \alpha) x$ for $x, y \in M$. Further, $x(G, \alpha) y$ and $y(G, \alpha) z$ give $x(G, \alpha) z$. For if $x$ not $(G, \alpha) z$, there is an $F(G, \alpha)$ set $F$ and a separation

$$
M-F=M_{x}+M_{z}, \quad x \in M_{x}, \quad z \in M_{z} .
$$

Now by our assumptions, $y$ can be in neither $M_{x}$ nor $M_{z}$ and hence must be 
in $F$. But this gives $y \in g \subset F$, where $g \in G$; and this is impossible since by $(G, \alpha)$-saturation, $g$ would have to contain both $x$ and $z$. This completes the proof.

(3.11) Corollary. Any $(G, \alpha)$-saturated collection $G$ in $M$ generates uniquely a collection $G^{\prime}$ of disjoint sets filling up $M$ and which is $(G, \alpha)$-determined.

(3.2) ThEOREM. The elements of any $(G, \alpha)$-determined collection $G^{\prime}$ in $M$ are disjoint closed sets.

For let $g^{\prime} \in G^{\prime}, y \in M-g^{\prime}$ and $x \in g^{\prime}$. Then $x$ not $(G, \alpha) y$ gives an $F(G, \alpha)$ set $F$ and a separation

$$
M-F=M_{x}+M_{y}, \quad M_{x} \supset x, \quad M_{y} \supset y .
$$

$(G, \alpha)$-coherence of $G^{\prime}$ gives $g^{\prime} \subset M_{x}+F$ so that $y$ is not a limit point of $g^{\prime}$. Accordingly $g^{\prime}$ is closed. Further, any element $g^{\prime \prime}$ of $G^{\prime}$ containing $y$ could not contain $x$; and since $x \in g^{\prime}$ was arbitrary, this gives $g^{\prime} \cdot g^{\prime \prime}=0$.

(3.21) Corollary. If $G$ is $(G, \alpha)$-saturated, the unique decomposition $G^{\prime}$ of $M$ generated by $G$ [see (3.11)] is a decomposition into disjoint closed sets. Further, if in addition $G$ is $(G, \alpha)$-coherent, $G$ will be a subcollection of $G^{\prime}$.

(3.3) ThEOREM. If $M$ is compact, any $(G, \alpha)$-determined collection $G^{\prime}$ in $M$ is upper semi-continuous [7].

For let $g_{1}^{\prime}, g_{2}^{\prime}, g_{3}^{\prime}, \ldots$ be a convergent sequence of elements of $G^{\prime}$ with limit set $L$ which contains a point $x$ of an element $g^{\prime}$ of $G^{\prime}$ and suppose there were a point $y \in L-L \cdot g^{\prime}$. By hypothesis there would exist an $F(G, \alpha)$ set $F$ and a separation

$$
M-F=M_{x}+M_{y}, \quad x \in M_{x}, \quad y \in M_{y} .
$$

But since $M_{x}$ and $M_{y}$ are open and each intersects $L$, for some $n$ we would have

$$
g_{n}^{\prime} \cdot M_{x} \neq 0 \neq g_{n}^{\prime} \cdot M_{y},
$$

contrary to the $(G, \alpha)$-coherence of $G^{\prime}$.

(3.31) Corollary. If $M$ is compact, any $(G, \alpha)$-saturated collection $G$ in $M$ generates uniquely $a(G, \alpha)$-determined upper semi-continuous decomposition $G^{\prime}$ of $M$.

Note. The elements of $G^{\prime}$ are therefore disjoint closed sets and $G^{\prime}$ is $(G, \alpha)$-dễermined.

(3.4) THEOREM. If $M$ is compact and $\alpha+\alpha=\alpha$, the elements of any $(G, \alpha)$ determined collection $G^{\prime}$ in $M$ such that $g \cdot g^{\prime} \neq 0$ implies $g \supset g^{\prime}$ or $g \subset g^{\prime}$ where $g \in G, g^{\prime} \in G^{\prime}$ are continua. 
For let $X \in G^{\prime}$. If $X$ is not connected there exists an open set $U$ such that

$$
U \cdot X \neq 0 \neq(M-U) \cdot X, \quad F(U) \cdot X=0,
$$

where $F(U)$ denotes the boundary $\bar{U}-U$ of $U$. Let $x \in U \cdot X$. For each $y \in F(U)$, there exists an $F(G, \alpha)$ set $E(y)$ and a separation

$$
M-F(y)=M(x)+M(y), \quad x \in M(x), \quad y \in M(y) .
$$

Since the sets $M(y)$ are open, there exists a finite set $y_{1}, y_{2}, \cdots, y_{k}$ in $F(U)$ such that $\sum_{1}^{k} M\left(y_{i}\right) \supset F(U)$. Let $V=U \cdot \prod_{1}^{k} M\left(x_{i}\right)$. Then since for each $i$, $y_{i} \cdot X=x \cdot E\left(y_{i}\right)=0$, it follows by the intersection condition on the $g$ and $g^{\prime}$ that $X \cdot \sum_{1}^{\mathbf{k}} F\left(y_{i}\right)=0$. Furthermore since $F(V) \subset \sum_{1}^{k} E\left(y_{i}\right)$, it follows that $\sum_{1}^{\mathfrak{k}} F\left(y_{i}\right)=F$ separates $x$ and $(M-U) \cdot X$. But since $\alpha+\alpha=\alpha, F$ is an $F(G, \alpha)$ and this contradicts the $(G, \alpha)$-coherence of $G^{\prime}$.

Note. Without the intersection condition on the $g$ and $g^{\prime}$ this result does not hold. For let $M$ be an interval $a b, G=\{x+a\}+\{x+b\}$ for all $x \in a b$ $-(a+b)$, and let $G^{\prime}$ consist of the single element $a+b$. Then $G^{\prime}$ is $(G, \alpha)$ determined but of course its one element is not connected.

(3.41) Corollary. If $M$ is compact, $\alpha+\alpha=\alpha, G^{\prime}$ is $(G, \alpha)$-determined and there exists a collection $H$ of disjoint sets in $M$ having both $G$ and $G^{\prime}$ as subcollections (in particular, if $G^{\prime} \supset G$ or if $G \supset G^{\prime}$ and the elements of $G$ are disjoint), then the elements of $G^{\prime}$ are continua.

(3.5) THEOREM. Let $G$ be any $(G, \alpha)$-determined collection of sets in a continuum $M$, where $\alpha=\omega, \aleph_{0}$ or $c$. The decomposition $G^{\prime}$ of $M$ generated by $G$ is a decomposition into continua and $G^{\prime} \supset G$ so that the elements of $G$ are necessarily continua. Thus also $G^{\prime}$ is $\left(G^{\prime}, \alpha\right)$-determined.

(3.6) Theorem. If $G^{\prime}$ is $(G, \alpha)$-determined, it is $\left(G^{\prime}, 1\right)$-coherent.

For if not, there exist two elements $X$ and $Y$ of $G^{\prime}$ and a separation

$$
M-X=M_{1}+M_{2}, \quad M_{1} \supset y_{1} \in Y, \quad M_{2} \supset y_{2} \in Y .
$$

Since $G^{\prime}$ is $(G, \alpha)$-saturated, there exists an $F(G, \alpha)$ set $F$ and a separation

$$
M-\dot{F}=M(x)+M\left(y_{2}\right), \quad M(x) \supset x \in X, \quad M\left(y_{2}\right) \supset y_{2} .
$$

But this gives the separation $M-F=\left(M_{1}+M(x)\right)+\left(M_{2} \cdot M\left(y_{2}\right)\right)$ with $y_{1} \in\left(M_{1}+M(x)\right), y_{2} \in M_{2} \cdot M\left(y_{2}\right)$ because $X-X \cdot F \subset M(x)$. This contradicts the $(G, \alpha)$-coherence of $G^{\prime}$.

(3.61) CoRollary. If $G$ is $(G, 1)$-saturated, the decomposition $G^{\prime}$ of $M$ generated by $G$ is $\left(G^{\prime}, 1\right)$-determined.

EXAMPLE. For $\alpha=4$, the collection $G^{\prime}$ generated by a $(G, 4)$-determined collection $G$ in a continuum $M$ may fail to be $\left(G^{\prime}, 4\right)$-coherent [although it must be $(G, 4)$-coherent, of course]. 
For let $M$ consist of $4 \operatorname{arcs} a x_{i} b, i=1,2,3,4$, joining two points $a$ and $b$ and otherwise disjoint, together with 8 arcs $a x_{i}$ and $x_{i} b, i=1,2,3,4$, intersecting each other and the arcs $a x_{i} b$ only in their indicated end points. Let $G$ be the collection of all individual points of $M-\left(a+b+x_{1}+x_{2}+x_{3}+x_{4}\right)$. Then $G$ is $(G, 4)$-determined; but the decomposition $G^{\prime}$ generated by $G$ has the additional elements $a+b, x_{1}, x_{2}, x_{3}$, and $x_{4}$ and clearly the sum of the last 4 elements separates $a$ and $b$ in $M$.

4. The case $\alpha=1$. The result just established suggests other important consequences in case the separation involved in our study is effected by a single element of the collection. Even though in this case $\alpha+\alpha$ is surely not equal to $\alpha$, nevertheless it will be shown that the elements of a $(G, \alpha)$-determined decomposition $G^{\prime}$ of a continuum $M$ will be continua provided the elements of $G$ are continua.

(4.1) Lemma. Let $M$ be a continuum and suppose $G$ is a collection of disjoint subcontinua of $M$. For any $p \in M$, the set $M_{p}$ of all $q \in M$ such that no element of $G$ separates $p$ and $q$ in $M$ is a continuum.

Proof. For any $x \in M-M_{p}$ there exists an $X \in G$ and a separation

$$
M-X=M_{p}(X)+M(X) \text {, where } M_{p}(X) \supset p, \quad M(X) \supset x .
$$

Since the sets $[M(X)], x \in M-M_{p}$, are open, a countable collection $\left[M\left(X_{i}\right)\right]$ of them covers $M-M_{p}$.

Let $N_{1}=M-M\left(X_{1}\right)$ and let $Y_{2}$ be the first member of the sequence $X_{2}, X_{3}, \cdots$ which is in $N_{1}$. Then $N_{2}=N_{1}-N_{1} \cdot M\left(Y_{2}\right)$ is a continuum, because $N_{1}$ is a continuum and

$$
N_{1}-Y_{2}=N_{1} \cdot M\left(Y_{2}\right)+N_{1} \cdot M_{p}\left(Y_{2}\right)
$$

is a separation. Let $Y_{3}$ be the first element of $\left[X_{i}\right]$ after $Y_{2}$ satisfying $Y_{3} \subset N_{2}$. Again $N_{3}=N_{2}-N_{2} \cdot M\left(Y_{3}\right)$ is a continuum. Continuing in this way we obtain a sequence of continua $N_{1} \supset N_{2} \supset N_{3} \supset \ldots$ such that

$$
\prod_{1}^{\infty} N_{i}=M-\sum_{1}^{\infty} M\left(Y_{i}\right), \text { where } Y_{1}=X_{1} \text {. }
$$

Now let $X_{n}$ be any set of $\left[X_{i}\right]$ not in $\left[Y_{i}\right]$. For some least $k$ we have $X_{n} \subset M\left(Y_{k}\right)$; and since $M_{p}\left(X_{n}\right)+X_{n} \supset M_{p}\left(Y_{k}\right)+Y_{k}$, we have $M\left(X_{n}\right) \subset M\left(Y_{k}\right)$. Therefore

and

$$
\sum_{1}^{\infty} M\left(Y_{i}\right)=\sum_{1}^{\infty} M\left(X_{i}\right)
$$

$$
M-\sum_{1}^{\infty} M\left(X_{i}\right)=\prod_{1}^{\infty} N_{i}=M_{p}
$$

Accordingly, $M_{p}$ is a continuum. 
Note. If $M$ is just connected and not compact, $M_{p}$ may be disconnected. For let $M$ be the graph of $y=\sin (1 / x)$ for $-1 \leqq x \leqq 1, x \neq 0$, together with the points $p=(0,0)$ and $q=(0,1)$, and let $G$ be the collection of single points of $M-(p+q)$. Then $M_{p}=p+q$.

(4.2) TheOREM. If $M$ and the elements of $G$ are continua and $G$ is $(G, 1)$ saturated, the elements of the upper semi-continuous decomposition $G^{\prime}$ generated by $G$ are continua. Further, $G^{\prime} \supset G$ and $G^{\prime}$ is $\left(G^{\prime}, 1\right)$-determined.

Since by $(3.1),(G, 1)$-conjugacy is an equivalence relation it follows that the decomposition $G^{\prime}$ of $M$ generated uniquely by $G$ as in (3.11) is identical with the decomposition of $M$ into distinct sets $M_{p}$ as defined in (4.1). Accordingly, the elements of $G^{\prime}$ are continua. Since the elements of $G$ are continua, $G$ is surely $(G, 1)$-coherent and hence by $(3.21), G^{\prime} \supset G$. Finally, that $G^{\prime}$ is $\left(G^{\prime}, 1\right)$-determined follows directly from $(3.6)$, because $G^{\prime}$ is $(G, 1)$-determined.

5. Generation of decompositions. The results established in the preceding two sections lead at once to the following principal theorem concerning the generation of a decomposition in a continuum by a given collection of sets.

(5.1) Theorem. Any $(G, \alpha)$-determined collection $G$ in a continuum $M$ generates uniquely an upper semi-continuous decomposition $G^{\prime}$ of $M$ containing $G$ which is $(G, \alpha)$-determined and $\left(G^{\prime}, 1\right)$-coherent. For $\alpha=\omega, \boldsymbol{N}_{0}$ or $c$ the elements of $G^{\prime}$ are necessarily continua, while for $\alpha=1$ this is true provided the elements of $G$ are continua.

Proof. Application of (3.11) and (3.21) gives the decomposition $G^{\prime}$ so that $G^{\prime} \supset G$ and $G^{\prime}$ is $(G, \alpha)$-determined. Since $M$ is compact, it follows by (3.3) that $G^{\prime}$ is upper semi-continuous. By $(3.6), G^{\prime}$ is $\left(G^{\prime}, 1\right)$-coherent. The final sentence in our theorem is a direct consequence of (3.5) and (4.2).

Note. For other values of $\alpha$, the last statement in the above theorem need not hold. For if $M$ is a $\theta$-curve consisting of three arcs $a x b, a y b$, and $a z b$ and $G$ is the collection of all individual points of $M-(a+b), G$ is $(G, 2)$-determined but $a+b$ is an element of the decomposition $G^{\prime}$ of $M$ generated by $G$. A similar example could be given for $\alpha$ any integer greater than 2 .

As a sort of converse, valid in any separable metric space $M$, we have

(5.2) TheOREM. Any upper semi-continuous decomposition $G$ of $M$ is $(G, c)$ saturated.

To prove this we have only to take, for any $g \in G$ and $p \in M-g$, an open set $U$ about $g$ not containing $p$, let $U_{0}$ be the sum of all elements of $G$ lying wholly in $U$, let $V$ be an open set satisfying $g \subset V \subset \bar{V} \subset U_{0}$, and finally let $F$ be the sum of all elements of $G$ intersecting $F(V)$. Then since $\bar{V} \subset U_{0}$ we have $F \subset U_{0} \subset U$ so that clearly $F$ separates $g$ and $p$. Further, upper semi-continuity of $G$ yields the fact that $F$ is closed. For $M-F$ consists of exactly the collec- 
tion of all elements of $G$ lying entirely in the open set $M-F(V)$. Accordingly $M-F$ is open and $F$ is closed. Finally, by definition $F$ is the sum of a collection of (surely not more than $c$ ) elements of $G$.

By similar reasoning we readily obtain

(5.3) If $M$ is regular or rational (that is, each point contained in arbitrary small neighborhoods with finite or countable boundaries, respectively), any upper semi-continuous decomposition $G$ of $M$ is $(G, \omega)$-saturated or $\left(G, \aleph_{0}\right)$ saturated respectively.

6. Equivalence of collections. Two collections $G_{1}$ and $G_{2}$ of subsets of $M$ will be called $\alpha$-equivalent provided that $\left(G_{1}, \alpha\right)$-conjugacy and $\left(G_{2}, \alpha\right)$-conjugacy are identical for all pairs $x, y \in M$, that is, provided $x\left(G_{1}, \alpha\right) y$ implies $x\left(G_{2}, \alpha\right) y$ and conversely for all $x, y \in M$. For example, if $M$ is the real number system, any two dense sets of single points in $M$ are $\alpha$-equivalent for all $\alpha$.

If $G_{1}$ and $G_{2}$ are $\left(G_{1}, \alpha\right)$-saturated and $\left(G_{2}, \alpha\right)$-saturated respectively, it is clear that $G_{1}$ and $G_{2}$ will be $\alpha$-equivalent if and only if they generate one and the same decomposition $G^{\prime}$ of $M$. Also, if $G_{1}$ and $G_{2}$ are $\left(G_{1}, \alpha\right)$-determined and $\left(G_{2}, \alpha\right)$-determined respectively and $G_{1}$ and $G_{2}$ are $\alpha$-equivalent, then by (3.21) both $G_{1}$ and $G_{2}$ are subcollections of this common decomposition $G^{\prime}$ of $M$.

(6.1) If $G_{1}$ is $\left(G_{1}, \alpha\right)$-determined and $G_{2}$ is any subcollection of $G_{1}$ which is $\alpha$-equivalent to $G_{1}$, then $G_{2}$ is $\left(G_{2}, \alpha\right)$-determined.

Since $G_{2} \subset G_{1}$ it follows that $G_{2}$ is $\left(G_{1}, \alpha\right)$-determined and thus is $\left(G_{2}, \alpha\right)$ coherent because $\left(G_{1}, \alpha\right)$-coherency implies $\left(G_{2}, \alpha\right)$-coherency. To see that $G_{2}$ is $\left(G_{2}, \alpha\right)$-saturated, let $x \in X \in G_{2}$ and suppose $x\left(G_{2}, \alpha\right) y$. Then by $\alpha$-equivalence of $G_{1}$ and $G_{2}$, we have $x\left(G_{1}, \alpha\right) y$; and by $\left(G_{1}, \alpha\right)$-saturation of $G_{2}$, this in turn implies $y \in X$.

For convenience of reference and for completeness, we state next a result proven in an earlier paper [1].

(6.2) THEOREM. If $M$ is connected, any $(G, 1)$-coherent collection $G$ of disjoint cuttings of $M$ contains a subcollection $Q$ which is $(Q, 1)$-determined and such that $G-Q$ is countable. Further, every element $X$ of $Q$ is a closed set, cuts $M$ irreducibly into just two components, and is of potential order 2 in $M$ relative to $Q$.

(6.3) Theorem. If $M$ is connected, any $(G, 1)$-coherent collection $G$ of disjoint cuttings of $M$ contains a countable subcollection $H$ which is 1-equivalent to $G$.

Proof. Obviously we need only consider the case where $G$ is uncountable. Let $Q$ be the subcollection of $G$ given by (6.2). Let $P=\sum_{1}^{\infty} p_{i}$ be a countable set dense in $M$; and for any pair of points $p_{i}, p_{j}$ of $P$ such that some element of $G$ separates $p_{i}$ and $p_{j}$ in $M$, let $G_{i j}$ be the set of all such elements if this set is countable and be a countable set of such elements whose sum is dense in 
the sum of all such elements otherwise. Let $H$ consist of all elements of all such $G_{i}$ thus chosen, together with all elements in the collection $G-Q$. Then clearly $H$ is a countable subcollection of $G$.

To show that $H$ is 1-equivalent to $G$, let $a, b \in M$ and suppose some element $g$ of $G$ separates $a$ and $b$ in $M$. If $g \in H$, there is nothing to prove. Otherwise, $g \in Q$; and since then $g$ is of potential order 2 relative to $Q$, there exist elements $X$ and $Y$ of $Q$ and a separation

$$
M-X-Y=M_{a}(X)+M_{b}(X) \cdot M_{a}(Y)+M_{b}(Y),
$$

where $M_{a}(X) \cdot M_{b}(Y)=0, M_{b}(X) \cdot M_{a}(Y) \supset g, M_{a}(X) \supset a, M_{b}(Y) \supset b$. There exist integers $i$ and $j$ such that $p_{i} \in M_{a}(X), p_{j} \in M_{b}(Y)$. Then by definition of $G_{i j}$, some element $Z$ of $G_{i j}$ lies in the open set $M_{b}(X) \cdot M_{a}(Y)$. This gives $Z \in H$, and since $M_{a}(Z) \supset M_{a}(X) \supset a+p_{i}, M_{b}(Z) \supset M_{b}(Y) \supset b+p_{j}$, where $M-Z=M_{a}(Z)+M_{b}(Z)$ is the separation generated by $Z, Z$ separates $a$ and $b$. 'Thus $a(H, 1) b$ implies $a(G, 1) b$ and, as the converse is obvious, the proof is complete.

(6.31) Corollary. Any $(G, 1)$-determined collection $G$ of cuttings of a connected set $M$ contains a countable $(H, 1)$-determined subcollection $H$ which is 1-equivalent to $G$.

For by (6.1) any subcollection $H$ of $G$ which is 1-equivalent to $G$ is $(H, 1)$ determined. Hence we have only to apply (6.3).

Known results [8] concerning local separating continua of a continuum $M$ (that is, continua $K$ in $M$ such that for some neighborhood $U$ of $K$ in $M$ there exists a separation of $\bar{U}-K$ between two points of the component of $\bar{U}$ containing $K$ ) yield at once:

(6.4) Theorem. Any uncountable collection $G$ of disjoint local separating continua of a continuum $M$ contains a $(Q, 2)$-determined subcollection $Q$ such that $G-Q$ is countable. Further, each element of $Q$ is of potential order 2 in $M$ relative to $Q$.

7. Existence theorems. It results at once from (5.2) that there always exist $(G, c)$-determined collections in any separable metric space. Indeed it is only necessary to let $G$ be the collection of all individual points of $M$ to obtain one example. For then clearly $G$ is $(G, 1)$-coherent and upper semicontinuous. However for other values of $\alpha$, the existence question does not lend itself to such simple handling. Our results in this connection are limited to locally connected sets $M$.

Since by an earlier theorem [1], any connected and locally connected set $M$ always contains an uncountable $(G, 1)$-coherent collection of cuttings of $M$, this fact together with (6.2) and (6.3) yields the following theorem.

(7.1) THEOREM. If $M$ is connected and locally connected, there exists a $(G, 1)$ - 
determined collection $G$ of subsets of $M$. Further, $G$ can be chosen either countable or uncountable.

(7.2) Theorem. If $M$ is a locally connected continuum and $F$ is any finite collection of disjoint subcontinua of $M$, there exists a $(G, \omega)$-determined collection $G$ of disjoint continua in $M$ containing $F$.

We first prove:

$\left({ }^{*}\right)$ If $F^{\prime}$ is any finite collection of disjoint continua in $M$ with sum $A$ and $\epsilon>0$ is arbitrary, there exists a finite collection $F^{\prime \prime}$ of disjoint continua in $M-A$ such that if $X \in F^{\prime}$ and $p \in M-V_{\epsilon}(X)$, then $p$ and $X$ are separated in $M$ by some subcollection of $F^{\prime \prime}$.

To prove $\left({ }^{*}\right)$, let $X \in F^{\prime}$ and $\sigma, \delta<\epsilon$ be so chosen that $\delta<\sigma, V_{3 \sigma}(X) \cdot(A-X)$ $=0$. By local connectedness of $M$, there are only a finite number of components of $\bar{V}_{\sigma}(X)-V_{\delta}(X)$ which contain boundary points of $V_{\delta}(X)$. Let $F_{x}^{\prime \prime}$ be the collection of these components and let $F^{\prime \prime}$ be the collection obtained by adding together all the elements of the $F_{x}^{\prime \prime}$ for all elements $X$ of $F^{\prime}$. Then clearly $F^{\prime \prime}$ satisfies all the conditions in $\left({ }^{*}\right)$.

Now to prove our theorem, take $F$ for $F^{\prime}$ and 1 for $\epsilon$ and apply $\left(^{*}\right)$, obtaining a collection $F_{1}$ for $F^{\prime \prime}$. Then take $F+F_{1}$ for $F^{\prime}, 1 / 2$ for $\epsilon$ and apply $\left(^{*}\right)$, obtaining a collection $F_{2}$ for $F^{\prime \prime}$. Next take $F+F_{1}+F_{2}$ for $F^{\prime}, 1 / 3$ for $\epsilon$ and again apply $\left(^{*}\right)$ obtaining $F_{3}$ for $F^{\prime \prime}$, and so on indefinitely. Finally, let $G$ denote the collection $F=\sum_{1}^{\infty} F_{n}$. Then $G$ is a collection of disjoint subcontinua of $M$. To show that $G$ is $(G, \omega)$-saturated, let $X \in G$ and $p \in M-X$. Then for $n$ sufficiently large we have $X \in F+\sum_{1}^{n} F_{i}$ and $1 / n<\rho(p, X)$. Accordingly, $F_{n+1}$ contains a set of elements separating $X$ and $p$ in $M$. Obviously $G$ is $(G, \omega)$ coherent and thus our proof is complete.

By essentially the same method the following result of greater generality is readily proven.

(7.3) ThEOREM. If $M$ is a locally connected continuum and $F$ is any closed subset of $M$, there exists $a(G, \omega)$-determined collection $G$ of disjoint continua in $M$ such that each component of $F$ is an element of $G$.

To see this we note first that, for any $\epsilon>0$, we can find a finite number of components $X_{1}, X_{2}, \cdots, X_{k}$ of $F$ and the same number of open sets $U_{1}, U_{2}, \ldots, U_{k}$ such that $X_{i} \subset U_{i} \subset V_{\epsilon}\left(X_{i}\right)$ for each $i, \bar{U}_{i} \cdot \bar{U}_{j}=0$ for $i \neq j$, and $F \subset \sum_{1}^{k} U_{i}$. Then for each $i$ we choose an open set $V_{i}$ such that $F \cdot U_{i} \subset V_{i}$ $\subset \bar{V}_{i} \subset U_{i}$, let $W_{i}$ be the (finite) collection of components of $\bar{U}_{i}-V_{i}$ which contain points of both $F\left(U_{i}\right)$ and $F\left(V_{i}\right)$ and let $W=\sum_{1}^{k} W_{i}$. Then for any component $X$ of $F$ and any $p \in M-V_{\epsilon}(X), p$ and $X$ are separated in $M$ by some (finite) subcollection of $W$. Thus we obtain a lemma essentially the same as $\left(^{*}\right)$ in the proof of (7.2); and if used in the same way, it yields our theorem.

By (5.1), the upper semi-continuous decomposition $G^{\prime}$ of $M$ generated by 
the collection $G$ in (7.2) or (7.3) is $\left(G^{\prime}, \omega\right)$-determined and is a decomposition into continua. Thus we have the following extension of a theorem of Roberts [9] and Moore [10].

(7.4) If $M$ is a locally connected continuum there exists $a\left(G^{\prime}, \omega\right)$-determined upper semi-continuous decomposition $G^{\prime}$ of $M$ into continua which contains as elements any given finite number of disjoint subcontinua of $M$ or indeed the components of any given closed subset of $M$.

In particular, any given finite number of points or the points in any given totally disconnected closed set in $M$ can be included as elements in such a decomposition $G^{\prime}$.

8. Mapping theorems. Since any upper semi-continuous decomposition of a space $M$ generates a continuous mapping on $M$ and since we have seen that $(G, \alpha)$-determined collections generate such decompositions, clearly it follows that the resulting mappings are completely generated by the original collections. Thus from (5.1) we have the following theorem.

(8.1) Theorem. Any $(G, \alpha)$-determined collection $G$ in a continuum $M$ generates uniquely a non-alternating transformation $f(M)=M^{\prime}$. For $\alpha=\omega, \boldsymbol{\aleph}_{0}$ or $c$, $f$ is monotone, while for $\alpha=1$ this is true provided the elements of $G$ are continua.

It may be recalled that a continuous transformation $f(A)=B$ is (a) monotone provided $f^{-1}(y)$ is a continuum for each $y \in B$, (b) non-alternating provided that for $x, y \in B$, no two points of $f^{-1}(x)$ are separated in $A$ by $f^{-1}(y)$.

Thus the conclusion in (8.1) that $f$ is non-alternating is equivalent to the fact that the decomposition $G^{\prime}$ of $M$ generated by $G$ is $\left(G^{\prime}, 1\right)$-coherent and the latter is given by (5.1).

The structure of the image space $M^{\prime}$ depends on that of $M$, on $\alpha$ and also on the relation of the elements of $G$ to the space $M$. In general it can be said that for $\alpha=1$, the image space $M^{\prime}$ will always be a dendrite, because any two points of $M^{\prime}$ are separated by a third point. Similarly for $\alpha=2, M^{\prime}$ will be a boundary curve (that is, a locally connected continuum every true cyclic element of which is a simple closed curve) because any two points of $M^{\prime}$ are separated by a pair of points. For similar reasons the case $\alpha=\omega$ will yield a regular curve for $M^{\prime}$ and $\alpha=\aleph_{0}$ yields a rational curve for $M^{\prime}$.

(8.2) THEOREM. In order that a continuum $M$ be mappable onto an interval by a monotone transformation it is necessary and sufficient that it contain an uncountable collection of disjoint connected cuttings.

To prove the sufficiency we note that under our hypothesis it follows by (6.2) that $M$ contains a $(Q, 1)$-determined collection of cuttings each of which is a continuum. Hence the mapping $f(M)=M^{\prime}$ generated by $Q$ under (8.1) is monotone and $M^{\prime}$ is a dendrite. Since $M^{\prime}$ can be monotone retracted into any simple $\operatorname{arc}$ in $M^{\prime}$, clearly there exists a monotone mapping $g\left(M^{\prime}\right)=I$ where 
$I$ is an interval. Then $g f(M)=I$ is our required monotone mapping.

On the other hand, if $f(M)=I=(a, b)$ is monotone, the collection $\left[f^{-1}(y)\right], a<y<b$, is an uncountable collection of disjoint continua each of which cuts $M$.

(8.3) THEOREM. In order that a continuum $M$ be mappable onto a circle by a monotone transformation it is necessary and sufficient that $M$ contain an uncountable collection of disjoint local separating continua no one of which cuts $M$.

For if $M$ contains such a collection $G$, by a previous theorem [8] there exist elements $A$ and $B$ of $G$, an uncountable subcollection $G_{1}$ of $G$, and a division of $M$ into two continua $H$ and $K$ such that $H \cdot K=A+B$, every element of $G_{1}$ lies in $H$ and separates $A$ and $B$ in $H$, and if $p \in H-(A+B)$ there are uncountably many elements of $G_{1}$ each of which separates $p$ and $A$ in $H$ and also uncountably many each separating $p$ and $B$ in $H$. By (6.2), $G_{1}$, considered as a collection in $H$, contains a $(Q, 1)$-determined subcollection $Q$ such that $G_{1}-Q$ is countable. By (5.1), $Q$ generates an upper semi-continuous decomposition $G^{\prime}$ of $H$ into continua such that $G_{1} \subset G^{\prime}$. Clearly $A$ and $B$ are elements of $G^{\prime}$. Now let $Q^{\prime}$ be the decomposition of $M$ into the set $K$ together with all elements of $G^{\prime}$ except $A$ and $B$. Clearly $Q^{\prime}$ is upper semicontinuous and the associated transformation $f(M)=M^{\prime}$ is monotone. Since $Q^{\prime}$ is $\left(Q^{\prime}, 2\right)$ determined in $M, M^{\prime}$ is a boundary curve. Further, since no element $X$ of $G_{1}$ cuts $M$ and hence $f(X)$ is a non-cut point of $M^{\prime}$, and also a non-end point of $M^{\prime}$ for each $X \in M^{\prime}, M^{\prime}$ has a true cyclic element, say $C$. Then if $r\left(M^{\prime}\right)=C$ is the monotone retraction of $M$ onto $C, r f(M)=C$ is a monotone mapping; and as $C$ is a simple closed curve, clearly this establishes our conclusion.

On the other hand, if $f(M)=C$ is monotone, where $C$ is a simple closed curve, $\left[f^{-1}(y)\right], y \in C$, gives the required collection of local separating continua of $M$.

From (7.4) we have at once (compare Roberts [9] and Moore [10]) the following theorem.

(8.4) THEOREM. If $F$ is any closed subset of a locally connected continuum $M$, there exists a monotone mapping $f(M)=K$ of $M$ into a regular curve $K$ such that each component $C$ of $F$ maps into a single point $y \in K$ and $C=f^{-1}(y)$.

Referring back to (8.2) and (8.3) we note in conclusion that if a continuum $M$ contains no uncountable collection of disjoint non-degenerate subcontinua, the collections mentioned in these theorems must reduce essentially to uncountable sets of cut points and local separating points respectively. Hence in particular we have the following theorem.

(8.5) TheOREM. In order that a rational continuum $M$ be monotone mappable onto the interval (circle) it is necessary and sufficient that it have uncountably many cut points (local separating points which are non-cut points). 


\section{Bibliography}

1. G. T. Whyburn, Non-separated cuttings of connected point sets, Trans. Amer. Math. Soc. vol. 33 (1931) pp. 444-454.

2. G. T. Whyburn, Non-alternating transformations, Amer. J. Math. vol. 56 (1934) pp. 294302.

3. D. W. Hall and G. E. Schweigert, Non-n-alternating transformations, Duke Math. J. vol. 3 (1937) pp. 623-626. 750.

4. J. F. Wardwell, Non-separating transformations, Duke Math. J. vol. 2 (1936) pp. 745-

5. A. D. Wallace, Concerning relatively non-alternating transformations, Proc. Nat. Acad. Sci. U.S.A. vol. 27 (1941) pp. 182-185.

6. P. A. White, On certain relatively non-alternating transformations, Bull. Amer. Math. Soc. abstract 46-5-347.

7. R. L. Moore, Concerning upper semi-continuous collections of continua, Trans. Amer. Math. Soc. vol. 27 (1925) pp. 416-428.

8. G. T. Whyburn, Local separating points of continua, Monatshefte für Mathematik und Physik vol. 36 (1929) pp. 305-314. See Theorem 10E.

9. J. H. Roberts, Concerning non-dense plane continua, Trans. Amer. Math. Soc. vol. 32 (1930) pp. 6-30.

10. R. L. Moore, Foundations of point set theory, Amer. Math. Soc. Colloquium Publications, vol. 13, 1932, p. 446.

UNIVERSITY OF VIRGINIA,

Charlottesville, Va. 